\title{
An Analytical Model for Prediction of Magnetic Flux Leakage from Surface Defects in Ferromagnetic Tubes
}

\author{
V. Suresh ${ }^{1}$, A. Abudhahir ${ }^{2}$ \\ ${ }^{1}$ National Engineering College, Department of Electronics and Instrumentation Engineering, Kovilpatti, Tamilnadu, India, \\ vseie@nec.edu.in \\ ${ }^{2}$ Vel Tech Multitech Dr.Rangarajan Dr.Sakunthala Engineering College, Department of Electrical and Electronics \\ Engineering, Chennai, India
}

\begin{abstract}
In this paper, an analytical model is proposed to predict magnetic flux leakage (MFL) signals from the surface defects in ferromagnetic tubes. The analytical expression consists of elliptic integrals of first kind based on the magnetic dipole model. The radial $\left(B_{z}\right)$ component of leakage fields is computed from the cylindrical holes in ferromagnetic tubes. The effectiveness of the model has been studied by analyzing MFL signals as a function of the defect parameters and lift-off. The model predicted results are verified with experimental results and a good agreement is observed between the analytical and the experimental results. This analytical expression could be used for quick prediction of MFL signals and also input data for defect reconstructions in inverse MFL problem.
\end{abstract}

Keywords: Analytical modeling, elliptic integrals, surface defect, dipolar magnetic charge.

\section{INTRODUCTION}

Magnetic Flux Leakage (MFL) technique is widely used for nondestructive evaluation of metal loss due to corrosion in underground oil and gas pipelines [1]-[2]. MFL technique is also used in testing of ferromagnetic tubes and other objects made from materials with high magnetic permeability [3]. In this technique, the test object is magnetized close to saturation flux density. The presence of a defect in the test object acts as localized magnetic dipole with effective magnetic moment opposite to the applied magnetic field. This results in a proportion of the magnetic field leak out of the object surface. This leakage flux is measured by magnetic sensors placed between the object and the magnetization arrangement. The sensors can measure axial $\left(B_{y}\right)$, radial $\left(B_{z}\right)$ or circumferential $\left(B_{x}\right)$ field components of leakage magnetic fields, although in practice only one component is usually measured [4].

Mathematical modeling enables a better understanding and effective utilization of the MFL technique. In general, two modeling methods, viz. analytical method and numerical method, are used to predict MFL signals from defects. In numerical method, the leakage field is obtained by solving the relevant Maxwell's equations with appropriate boundary conditions. Numerical method is capable of modeling of nonlinear problems and complicated geometries. However, computation time is high and also requires domain experts for further processing. In analytical method, defects are assumed as magnetic dipoles developed at the walls of the defect. The MFL signals are calculated from the magnetic field of the magnetic dipoles. The analytical modeling offers closed form solution and simple analysis of the defect region. Zatsepin and Shcherbinin [5] pioneered the dipole modeling of the MFL technique for determination of leakage fields from two-dimensional (2D) surface-breaking defects. They proposed that MFL signal arises from induced magnetic polarization at the walls of a defect. They approximated 2D defects as line dipoles of constant magnetic charge density and derived the expressions for tangential and normal components of leakage magnetic fields due to the defect. Förster [6] derived analytical expression for the induced surface charge density of surface defects with rectangular cross-sections by accounting the magnetic properties of the material and the applied magnetizing field strength. Zhang et al. [7] proposed an analytical expression for two kinds of sub-surface defects, such as rectangular defects and elliptical defects, while considering the magnetic image effects from the spatial boundary and the defect boundary. Mandache and Clapham developed an analytical model based on dipolar magnetic charge to determine the larger length of surface breaking defects in ferromagnetic steel plate. Four different types of geometry were experimentally investigated and they validated the analytical model with the experimental results. Constant lift-off is considered and the lift-off studies have 
not been performed [8]. Dutta et al. proposed an analytical model by accounting the variation of surface magnetic charge density for defect surfaces oblique to the direction of applied field. The model was able to predict all the orthogonal components of 3D-MFL fields of a surfacebreaking defect [9]. They also proposed that the use of the tangential (circumferential) component of MFL signal would be useful for determination of location of defects with respect to the sensor [10]. Magnetic leakage profiles of the interacting and non-interacting defects are analyzed and we conclude that the center to center distance between two axially aligned defects is greater than the four times of the defect radius is considered as non-interacting defect. The non-interacting defect has no flux shielding effect [11]. John T. Conway derived the formula for the magnetic field components of the coil of rectangular cross section. Radial and Axial leakage profile of the circular defect can be estimated using the elliptical integral method [12].

In this paper, a new analytical expression in terms of complete elliptic integrals of first kind is proposed to predict the radial leakage fields $\left(B_{z}\right)$ of the cylindrical surface defects in ferromagnetic tube. The effectiveness of the model has been studied by analyzing MFL signals as a function of the defect parameters and lift-off. The diameter of the hole is determined from the MFL profile predicted by the analytical expression. Performance of the proposed analytical expression is considered for the non-interacting defect. The correct estimation of the length between the two centers of the defect has shown an efficient performance of the proposed analytical model. Finally, the validation of the model with the experimental measurement using Hall sensor is discussed.

\section{ANALYTICAL MODEL FOR LEAKAGE FIELD}

In this section, a simple analytical dipole model is developed to predict the MFL signals of a surface defect on the ferromagnetic material. Consider a cylindrical surface defect on the surface of a ferromagnetic tube (shown in Fig.1.).

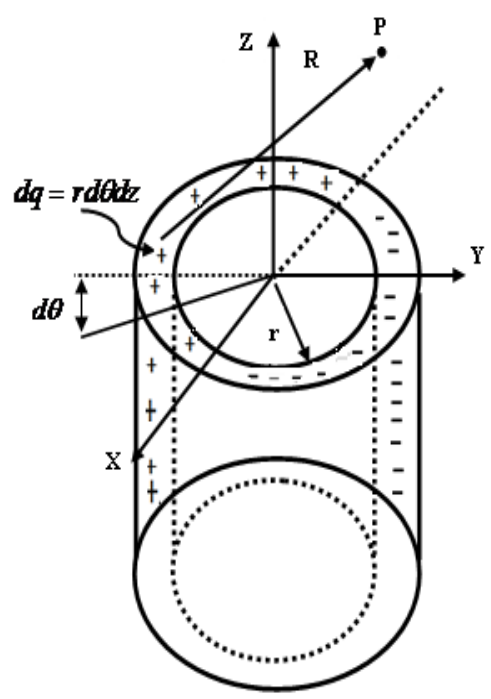

Fig.1. Cylindrical hole with dipole.
The radius of the defect is ' $r$ ' and depth ' $b$ '. The center of the defect is considered to be the origin. The magnetic field is assumed to be applied in the ' $y$ ' direction. The $B_{z}$ component of MFL signal is predicted at an arbitrary point $P$ located at a lift-off of $h$. The analytical modeling is complicated by means of non-linear characteristic of material and hence, while deriving the analytical expression, variations in magnetization and permeability variations are avoided by assuming high magnetic field.

According to dipole modeling, the opposite magnetic polarities appear on the wall of the cylinder. The half of the cylindrical defect develops the north polarity with the magnetic charge density $+\sigma$ and the other half of the defect develops the south polarity with the magnetic charge density $-\sigma$. In this model, we neglect the variation of $\sigma$ along the depth of the defect. Consider a small magnetic charge element $d q$ located at the co-ordinates $(\mathrm{r} \cos \theta, \mathrm{r} \sin \theta, \mathrm{z})$. Then, the magnetic charge $d p$ of the surface element is given by

$$
d p=\sigma \sin \theta d q
$$

The differential charge element $d q$ can be written as

$$
d q=r d \theta d z
$$

For $2 \mathrm{D}$ axi-symmetry defects, $\theta=90^{\circ}$. Then the magnetic field at a point in the ' $y z$ ' plane may be written as

$$
d \vec{B}=\frac{d p}{4 \pi R^{3}} \vec{R}
$$

Where $R$ is the distance from the small magnetic charge element to the field computing point $\mathrm{P}$. The distance from the North Pole to the point $\mathrm{P}$ is given by

$$
R_{+}=\sqrt{r^{2} \cos ^{2} \theta+\left(y+r^{2} \cos ^{2} \theta\right)+(h-z)^{2}}
$$

The distance from the South-pole to the point is given by

$$
R_{-}=\sqrt{r^{2} \cos ^{2} \theta+\left(y-r^{2} \cos ^{2} \theta\right)+(h-z)^{2}}
$$

Combining the equations (1) and (3), the magnetic field density can be written as

$$
d \vec{B}=\frac{\operatorname{\sigma rd} \theta d z}{4 \pi R^{3}} \vec{R}
$$

The radial leakage field can be written as

$$
d B_{Z}^{+}=\frac{\sigma r d \theta d z}{4 \pi R_{+}^{3}}(h-z)
$$

The axial leakage field can be written as 


$$
d B_{z}^{+}=\frac{\sigma r d \theta d z}{4 \pi R_{+}^{3}}(y+r \sin \theta)
$$

The defect geometry considered here is of $2 \mathrm{D}$ axisymmetry and hence the $\mathrm{x}$-component vanishes. The dipole modeling of radial leakage field is obtained by double integrating the equation. The integrations are applied over 0 to $\pi$ and $-\mathrm{b}$ to 0 to obtain the total leakage field.

$$
\begin{aligned}
B_{z}^{+} & =\frac{\sigma r}{4 \pi} \int_{0}^{\pi} d \theta \int_{-b}^{0} \frac{(h-z) d z}{\left(r^{2} \cos ^{2} \theta+(y+r \sin \theta)^{2}+(h-z)^{2}\right)^{\frac{3}{2}}} \\
& =\frac{\sigma r}{4 \pi} \int_{0}^{\pi} d \theta \int_{-b}^{0} \frac{(h-z) d z}{\left(r^{2}+y^{2}+2 r y \sin \theta+(h-z)^{2}\right)^{\frac{3}{2}}}
\end{aligned}
$$

First, the integration with respect to $d z$ is solved.

Let $h-z=u$ and $r^{2}+y^{2}+2 r y \sin \theta=a^{2}$

Then, $d z=-d u$; When $z=0, u=h$; When $z=-b, u=h+b$

$$
\begin{aligned}
& \int_{-b}^{0} \frac{(h-z) d z}{\left(r^{2}+y^{2}+2 r y \sin \theta+(h-z)^{2}\right)^{\frac{3}{2}}} \\
& =\int_{h+b}^{h} \frac{-u d u}{\left(a^{2}+u^{2}\right)^{\frac{3}{2}}} \\
& =\left[\frac{1}{\sqrt{a^{2}+h^{2}}}-\frac{1}{\sqrt{a^{2}+(h+b)^{2}}}\right]
\end{aligned}
$$

Using equation (10) in equation (9), we get

$$
\begin{aligned}
& B_{z}^{+}=\frac{\sigma r}{4 \pi} \int_{0}^{\pi} \frac{d \theta}{\sqrt{r^{2}+y^{2}+2 r y \sin \theta+h^{2}}} \\
& -\frac{\sigma r}{4 \pi} \int_{0}^{\pi} \frac{d \theta}{\sqrt{r^{2}+y^{2}+2 r y \sin \theta+(h+b)^{2}}}
\end{aligned}
$$

The integral of the first kind in equation (11) has been solved as,

$$
\begin{gathered}
\frac{\sigma r}{4 \pi} \int_{0}^{\pi} \frac{d \theta}{\sqrt{r^{2}+y^{2}+2 r y \sin \theta+h^{2}}} \\
=\int_{0}^{\pi} \frac{d \theta}{\sqrt{\left(r^{2}+y^{2}+h^{2}\right)\left(1+\frac{2 r y \sin \theta}{r^{2}+y^{2}+h^{2}}\right)}}
\end{gathered}
$$

Equation (12) becomes

$$
\begin{aligned}
& \int_{0}^{\pi} \frac{d \theta}{\sqrt{\left(r^{2}+y^{2}+h^{2}\right)\left(1+k^{2} \sin \theta\right)}} \\
& =\frac{1}{\sqrt{\left(r^{2}+y^{2}+h^{2}\right)}} \int_{0}^{\pi} \frac{d \theta}{\sqrt{1+k^{2} \sin \theta}} \\
& =\frac{k}{\sqrt{2 r y}} \frac{4}{\sqrt{1+k^{2}}} k\left(\sqrt{\frac{2 k^{2}}{1+k^{2}}}\right)
\end{aligned}
$$

Where $k$ is the elliptic integral of first kind.

Similarly, the integral of second kind in equation (11) can be solved to yield as,

$$
\begin{aligned}
& \int_{0}^{\pi} \frac{d \theta}{\sqrt{r^{2}+y^{2}+2 r y \sin \theta+(h+b)^{2}}} \\
& =\frac{l}{\sqrt{2 r y}} \frac{4}{\sqrt{1+l^{2}}} k\left(\sqrt{\frac{2 l^{2}}{1+l^{2}}}\right)
\end{aligned}
$$

Where, $l^{2}=\frac{2 r y}{r^{2}+y^{2}+(h+b)^{2}}$,

Thus, the radial magnetic leakage flux due to the northpole is given by,

$$
\begin{aligned}
& B_{z}^{+}=\frac{\sigma r}{4 \pi} \frac{k}{\sqrt{2 r y}} \frac{4}{\sqrt{1+k^{2}}} k\left(\sqrt{\frac{2 k^{2}}{1+k^{2}}}\right) \\
& -\frac{\sigma r}{4 \pi} \int_{0}^{\pi} \frac{l}{\sqrt{2 r y}} \frac{4}{\sqrt{1+l^{2}}} k\left(\sqrt{\frac{2 l^{2}}{1+l^{2}}}\right)
\end{aligned}
$$

The radial leakage field due to south-pole can be written as

$$
B_{z}^{-}=-\frac{\sigma r}{4 \pi} \int_{0}^{\pi} d \theta \int_{-b}^{0} \frac{(h-z) d z}{\left(r^{2} \cos ^{2} \theta+(y-\sin \theta)^{2}+(h-z)^{2}\right)^{\frac{3}{2}}}
$$

By solving the above equation, the radial magnetic leakage flux due to the south-pole is given by, 


$$
\begin{aligned}
& B_{z}^{-}=-\frac{\sigma r}{4 \pi} \frac{2}{\sqrt{(r-y)^{2}+h^{2}}} \frac{1}{\sqrt{1-m}} k\left(\frac{-m}{1-m}\right) \\
& +\frac{\sigma r}{4 \pi} \frac{2}{\sqrt{(r-y)^{2}+(h+b)^{2}}} \frac{1}{\sqrt{1-n}} k\left(\frac{-n}{1-n}\right)
\end{aligned}
$$

Where

$$
\begin{gathered}
m^{2}=\frac{4 r y}{(r-y)^{2}+h^{2}} \\
n^{2}=\frac{4 r y}{(r-y)^{2}+(h+b)^{2}}
\end{gathered}
$$

Hence, the total radial leakage field due to cylindrical surface defect can be expressed as,

$$
\begin{gathered}
B_{z}=B_{z}^{+}+B_{z}^{-} \\
B_{z}=\frac{\sigma r}{4 \pi} \frac{k}{\sqrt{2 r y}} \frac{4}{\sqrt{1+k^{2}}} k\left(\sqrt{\frac{2 k^{2}}{1+k^{2}}}\right) \\
-\frac{\sigma r}{4 \pi} \int_{0}^{\pi} \frac{l}{\sqrt{2 r y}} \frac{4}{\sqrt{1+l^{2}}} k\left(\sqrt{\frac{2 l^{2}}{1+l^{2}}}\right) \\
-\frac{\sigma r}{4 \pi} \frac{2}{\sqrt{(r-y)^{2}+h^{2}}} \frac{1}{\sqrt{1-m}} k\left(\frac{-m}{1-m}\right) \\
+\frac{\sigma r}{4 \pi} \frac{2}{\sqrt{(r-y)^{2}+(h+b)^{2}}} \frac{1}{\sqrt{1-n}} k\left(\frac{-n}{1-n}\right)
\end{gathered}
$$

The final obtained expression comprises complete elliptic integrals of first kind ( $k, \mathrm{l}, \mathrm{m} \& \mathrm{n})$. The solution for the equation (19) is obtained using MATLAB software. The same procedure is adapted for obtaining the radial leakage field expression for the interacting holes. In case of noninteracting hole, one is centered at $\left(0, y^{+} j, 0\right)$ and the other hole is centered at $(0, y-j, 0)$.The appropriate substitution has to be made to obtain the analytical expression.

\section{RESULTS AND DISCUSSION}

\section{A. MFL signals for single holes}

Fig.2. shows the model predicted radial leakage profile for a single hole of $16 \mathrm{~mm}$ diameter and $3 \mathrm{~mm}$ depth at $1.5 \mathrm{~mm}$ lift-off. The values are normalized with respect to the corresponding maximum amplitude, since we are not concerned about the maximum leakage field amplitude.

The peak-to-peak distance of the analytically obtained radial leakage component is used for the evaluation of defect diameter. From the graph it is observed that the peak-to-peak distance between the normalized radial MFL values is $16.10 \mathrm{~mm}$. Percentage of the error for this single cylindrical hole is $0.62 \%$. The diameter estimated using analytical expression proposed by Mandache and Clapham [8] was $15.7 \mathrm{~mm}$ whose percentage error is $1.87 \%$. Proposed analytical expression has minimum error percentage.

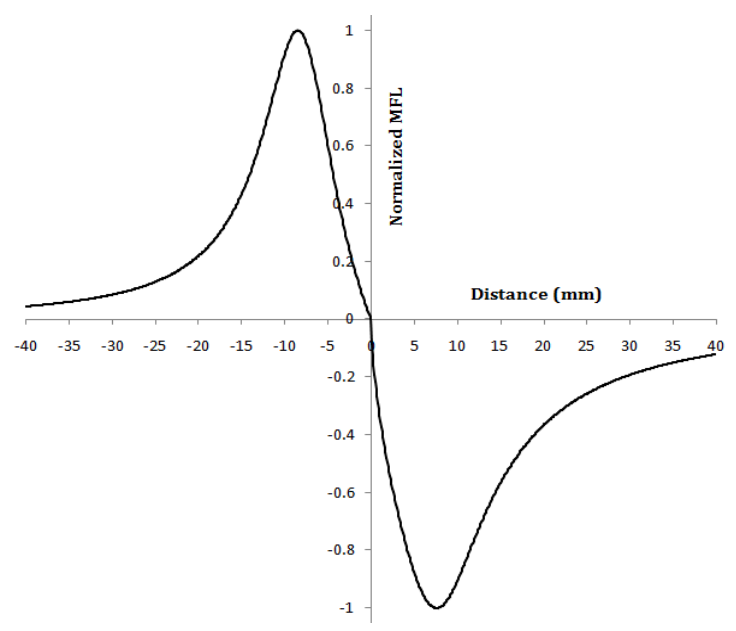

Fig.2. Radial leakage profile for a hole of $16 \mathrm{~mm}$ diameter.

Table 1. Percentage of error for different diameter estimation using proposed model.

\begin{tabular}{|c|c|c|}
\hline $\begin{array}{c}\text { Original } \\
\text { Diameter } \\
(\mathrm{mm})\end{array}$ & $\begin{array}{c}\text { Obtained } \\
\text { Diameter } \\
(\mathrm{mm})\end{array}$ & $\begin{array}{c}\text { Error } \\
(\%)\end{array}$ \\
\hline 22 & 21.8 & 0.9 \\
\hline 20 & 19.8 & 1.0 \\
\hline 18 & 17.9 & 0.5 \\
\hline 16 & 16.1 & 0.6 \\
\hline 14 & 14.3 & 2.1 \\
\hline 12 & 12.5 & 4.1 \\
\hline 10 & 10.8 & 8.0 \\
\hline 8 & 9.3 & 16.2 \\
\hline 6 & 8.0 & 33.3 \\
\hline
\end{tabular}

Table 2. Percentage of error for different lift-off.

\begin{tabular}{|l|l|l|l|}
\hline $\begin{array}{l}\text { Lift off } \\
(\mathrm{mm})\end{array}$ & $\begin{array}{l}\text { Original } \\
\text { diameter } \\
(\mathrm{mm})\end{array}$ & $\begin{array}{l}\text { Obtained } \\
\text { diameter } \\
(\mathrm{mm})\end{array}$ & $\begin{array}{l}\text { Error } \\
(\%)\end{array}$ \\
\hline 1.5 & 6 & 8 & 33.3 \\
\hline 1.0 & 6 & 7.1 & 25.0 \\
\hline 0.5 & 6 & 7 & 16.6 \\
\hline 0.25 & 6 & 6.9 & 15.0 \\
\hline 0.1 & 6 & 6.8 & 13.3 \\
\hline
\end{tabular}

Table 1. shows the error percentage for different diameter of $3 \mathrm{~mm}$ depth hole while considering the lift-off distance, which is distance between the surface defect and field calculation point and is $1.5 \mathrm{~mm}$. The results show that the proposed analytical modeling provides good results for larger diameter. However, in the case of smaller diameter the error percentage is high. Hence, further studies have been carried out to reduce the error percentage for smaller diameter defects. The lift-off variation in the magnetic flux leakage inspection has varying amplitude and base line magnitude. 
Lift-off studies have been carried out for cylindrical hole of $6 \mathrm{~mm}$ diameter and 3mm depth. The results are shown in Table 2. Lower lift-off value has minimum error percentage. For further studies in this paper the lift-off is considered as $0.1 \mathrm{~mm}$.

\section{B. MFL signals for non-interacting holes}

According to the code z66e of 'oil and gas pipe line system' the non-interacting defect is defined as the distance between the two defects which is large longitudinal length of the diameter of the defect [11]. In this work, two different cases are considered. In the first case, the distance between the two centers of holes is five times the diameter of a hole. The second case deals with two holes of different diameters; diameter of hole 1 is $5 \mathrm{~mm}$ and hole 2 is $10 \mathrm{~mm}$. The distance between the centers of the two holes is $32 \mathrm{~mm}$. For both cases constant depth $1 \mathrm{~mm}$ and lift-off $0.1 \mathrm{~mm}$ is considered. Table 3. describes the dimension of the two cases of non-interacting holes.

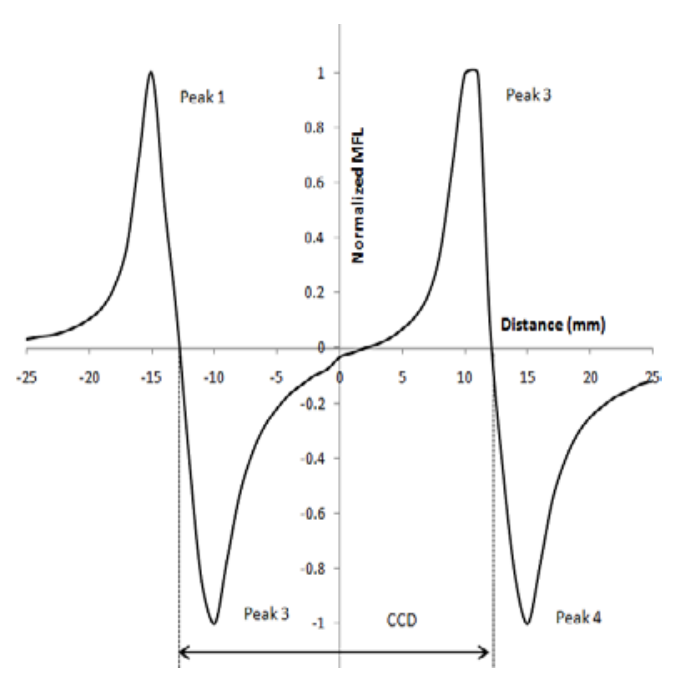

a)

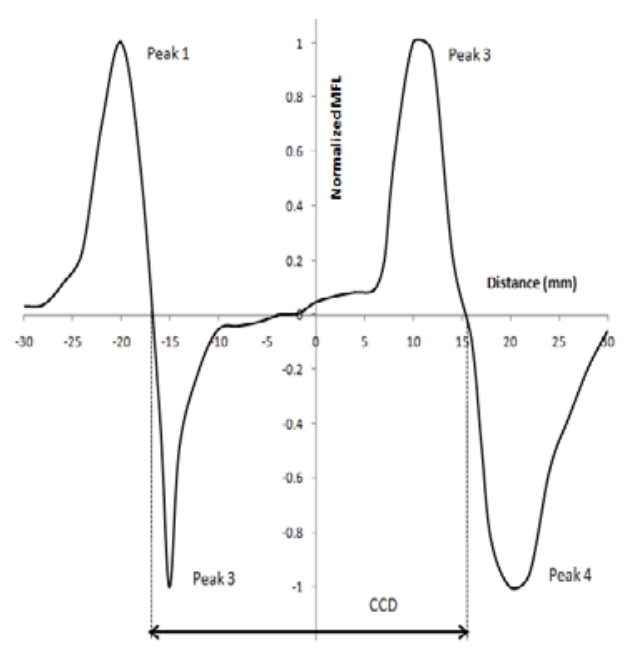

b)

Fig.3. Normalized radial MFL signals for interacting holes a) case -i b) case-ii.
Fig.3.a) and Fig.3.b) show the normalized MFL signals of the non-interacting holes. It is observed that the Center to Center Distance between the holes (CCD) for two cases is precisely reproduced in the proposed analytical model. The diameter of hole 1 is measured as the distance between peak 1 and peak 2 . The diameter of hole 2 is measured as the distance between peak 3 and peak 4 . From the graph it is clear that the analytical modeling predicts well the diameter of non-interacting holes. The separation of peak positions is nearly matching with the hole-to-hole separations.

Table 3. Dimensions for non-interacting holes.

\begin{tabular}{|l|l|l|l|}
\hline & $\begin{array}{c}\text { First } \\
\text { hole } \\
\text { Diameter }\end{array}$ & $\begin{array}{c}\text { Second } \\
\text { hole } \\
\text { Diameter }\end{array}$ & $\begin{array}{c}\text { Center to Center } \\
\text { Distance } \\
\text { between the } \\
\text { holes (CCD) }\end{array}$ \\
\hline Case (i) & $5 \mathrm{~mm}$ & $5 \mathrm{~mm}$ & $25 \mathrm{~mm}$ \\
\hline Case (ii) & $5 \mathrm{~mm}$ & $10 \mathrm{~mm}$ & $32 \mathrm{~mm}$ \\
\hline
\end{tabular}

\section{Comparison of analytical and experimental results}

MFL experiment has been carried out on the Modified 9Cr- 1Mo steam generator (SG) tube used in Power Plants. An artificial cylindrical hole of $5 \mathrm{~mm}$ radius and $2.3 \mathrm{~mm}$ depth was machined on the SG tube (length $1000 \mathrm{~mm}$, outer diameter $17.4 \mathrm{~mm}$ and wall thickness $2.3 \mathrm{~mm}$ ) by electrodischarge machining (EDM) process.

A Hall sensor (CYSH12AF) of indium stibnite (InSb) semiconductor supplied by Cheng yang Technologies, Germany was used. Agilent make Programmable DC regulated power supply (U8002A) was used as power source to the magnetizer coil. Multi Function Calibrator / Meter (U1401B) was used as current source for the Hall sensor. National Instruments (NI) product, PCI 7352 Motion Controller and MID-7602- 2 Axis Stepper Motor Drive were utilized to achieve the performance of the automated probe pulling mechanism. The output of the Hall sensor was acquired by means of NI DAQ 9207 into the personal computer. Programs were developed in LabVIEW 2011 for automation of the probe pulling mechanism and data acquisition of the Hall sensor output.

The automatic probe pulling mechanism to scan the SG tube is shown in Fig.4. This system helps to perform forward and reverse linear scanning of the SG tube of one meter length with a resolution of $1 \mathrm{~mm} / \mathrm{step}$. The linear movement is achieved by means of sprocket - chain mechanism which is controlled by NI micro-step stepper motor, motion control card - PCI 7352, MID driver - 9602 and two-axis stepper motor drive. Fig.5. shows the comparison of normalized MFL values of proposed analytical and experimental results. As can be seen, a good agreement is observed between the analytical and the experimental results.

The proposed analytical expression is simple and it does not require expensive software. It could be used for quick prediction of MFL signals to create a large database for testing several solution methods of defect reconstructions in inverse MFL problem. 


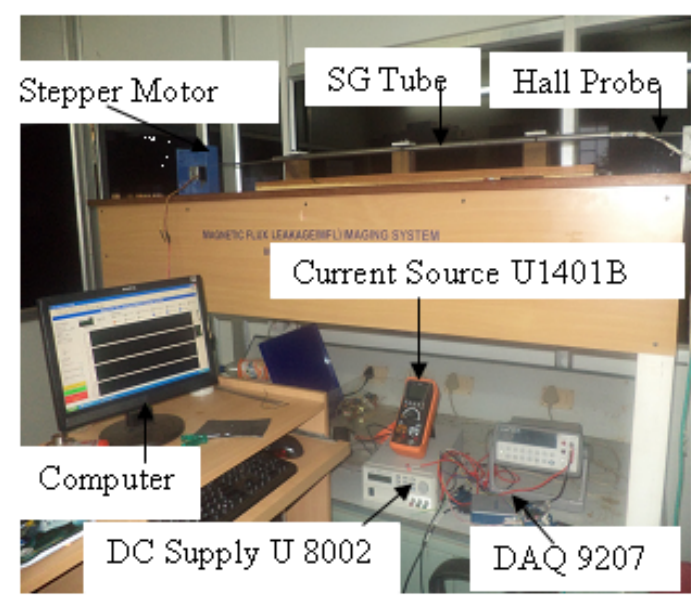

Fig.4. Automatic probe pulling setup.

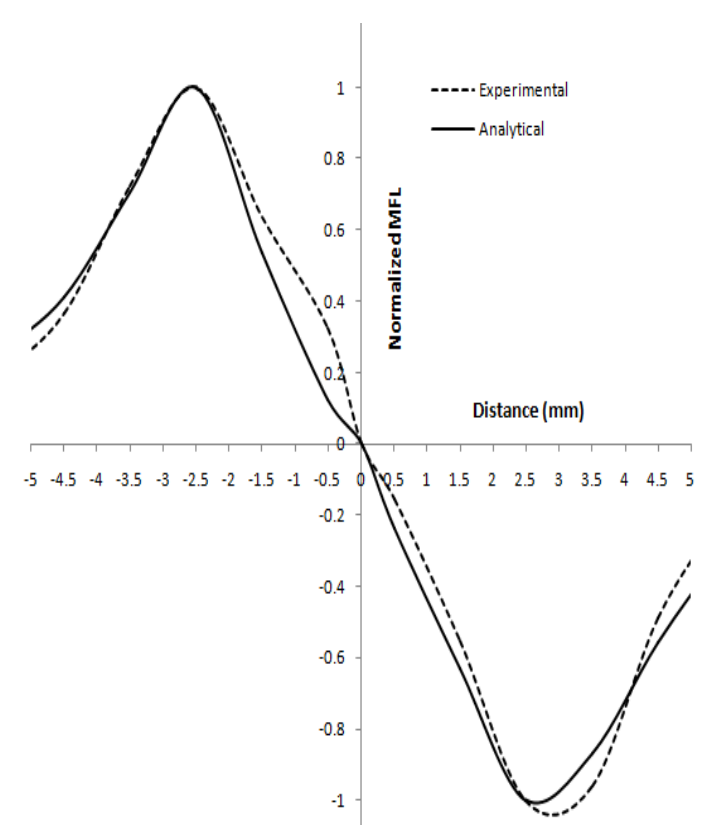

Fig.5. Comparison of experimental and analytical results.

\section{CONCLUSION}

An analytical model consisting of elliptic integrals of first kind is proposed to predict MFL signals from the surface defects in ferromagnetic tubes. Comparison between the earlier reported works with the proposed work has positive increment. Good correlation among the experimental and proposed expression shows the efficiency of the derived analytical expression. Lift-off plays a vital role to detect the defects in the tubes. Smaller lift-off has capacity to detect the smaller diameter defect. Non-interacting holes are identified at correct location by means of the proposed expression. The proposed expression could be used for quick prediction of MFL signals from surface defects in ferromagnetic tubes and pipes and this data could be used for defect reconstructions in inverse MFL problem.

\section{ACKNOWLEDGMENT}

The authors would like to thank Dr. B. Purna Chandra Rao, Head NDE Division \& Head, Robotics, Prof. HBNI, IGCAR, Kalpakkam, India and Dr.W. Sharatchndra Singh, SO/D, Electromagnetic Measurements \& Structural Integrity Section, IGCAR, Kalpakkam, India for their technical support

\section{REFERENCES}

[1] Lukyanets, S., Snarskii, A., Shamonin, M., Bakaev, V. (2003). Calculation of magnetic leakage field from a surface defect in a linear ferromagnetic material: An analytical approach. NDT \& E International, 36, 5155.

[2] Lijian Yang, Guoguang Zhang, Gang Liu, Songwei Gao. (2008). Effect of lift-off on pipeline magnetic flux leakage inspection. In 17th World Conference on Nondestructive Testing, 25-28 October, 2008, Shanghai, China.

[3] Snarskii, A.A., Zhenirovskyy, M., Meinert, D., Schulte, M. (2010). An integral equation model for the magnetic flux leakage method. NDT \& $E$ International, 43, 343-347.

[4] Rao, B.P.C. (2012). Magnetic flux leakage testing: Basics. Journal of Non-Destructive Testing \& Evaluation, 11 (3), 7-19.

[5] Zatsepin, N.N., Shcherbinin, V.E. (1966). Calculation of the magnetostatic field of surface defects. I. Field topography of defect models. Defektoskopiya, 5, 5059.

[6] Forster, F. (1986). New findings in the field of non destructive magnetic leakage field inspection. NDT \& E International, 19 (1), 3-14.

[7] Zhang, Y., Sekine, K., Watanabe, S. (1995). Magnetic leakage field due to sub-surface defects in ferromagnetic specimens. NDT \& E International, 28 (2), 67-71.

[8] Mandache, C., Clapham, L. (2003). A model for magnetic flux leakage signal predictions. Journal of Physics D: Applied Physics, 36, 2437-2421.

[9] Dutta, S.M., Ghorbel, F.H., Stanley, R.K. (2009). Dipole modeling of magnetic flux leakage. IEEE Transactions on Magnetics, 45 (4), 1959-1965.

[10] Dutta, S.M., Ghorbel, F.H., Stanley, R.K. (2009). Simulation and analysis of 3-D magnetic flux leakage. IEEE Transactions on Magnetics, 45( 4), 1966-1972.

[11] Mandache, C., Shiari, B., Clapham, L. (2005). Defect separation considerations in magnetic flux leakage inspection. Insight, 47 (5), 269-273.

[12] Conway, J.T. (2006). Trigonometric Integrals for the magnetic field of the coil of rectangular cross section. IEEE Transactions on Magnetics, 42 (5), 1538-1548.

Received October 6, 2015. Accepted January 28, 2016. 